Supporting information for manuscript

\title{
Thermoplastic PLA-LCP composites: a route towards sustainable, reprocessable, and recyclable reinforced materials.
}

Gijs W. de Kort*, Lucienne Bouvrie, Sanjay Rastogi, Carolus H.R.M Wilsens*

Aachen-Maastricht Institute for Biobased Materials (AMIBM), Faculty of Science and Engineering, Maastricht University, Urmonderbaan 22, 6167 RD, Geleen, The Netherlands.

*Corresponding author: gijs.dekort@ maastrichtuniversity.nl; karel.wilsens@ maastrichtuniversity.nl KEYWORDS: mechanical reprocessing, thermotropic polyester, blend, morphology

Supporting Information: 10 pages, 6 Figures 


\section{WAXD-diffractograms and orientation parameter as function of matrix $M_{\mathrm{w}}$.}

All diffractograms, with the exception of PLA-122 (30 wt\%) and $\mathrm{R}_{0}(0 \mathrm{wt} \%)$, consist of two superimposed signals (Figure S1, top): an isotropic amorphous halo, corresponding to the PLA phase, and two amorphous arcs, corresponding to the oriented LCP phase (as marked in PLA-245 $(30 \mathrm{wt} \%))$. The magnitude of the color difference between both signals is an indication for the level of LCP orientation: a larger difference in color between the two signals (PLA-245 (30 wt\%)) indicates, whereas a smaller color difference signifies a slightly lower level of orientation (PLA$153(30 \mathrm{wt} \%))$. The diffractograms of PLA-122 (30 wt\%) and $\mathrm{R}_{0}(0 \mathrm{wt} \%)$ both show an isotropic amorphous halo. In however, in the case PLA-122 (30wt\%), the highest signal intensity is obtained at higher diffraction angles. This is due the overlap between the signals of both the isotropic components, and the LCP having a signal at a larger diffraction angle. The diffractogram of $\mathrm{R}_{7}(30$ $w t \%)$ shows two additional faint arcs at a lower angle (towards the center of the image), which indicates the presence of a small amount of oriented PLA crystallites. This change crystallization behavior of the PLA can be attributed either to enhanced nucleation resulting from the build up of impurities, or to the presence of some fraction of non-linear chains, as indicated in Figures S2. 

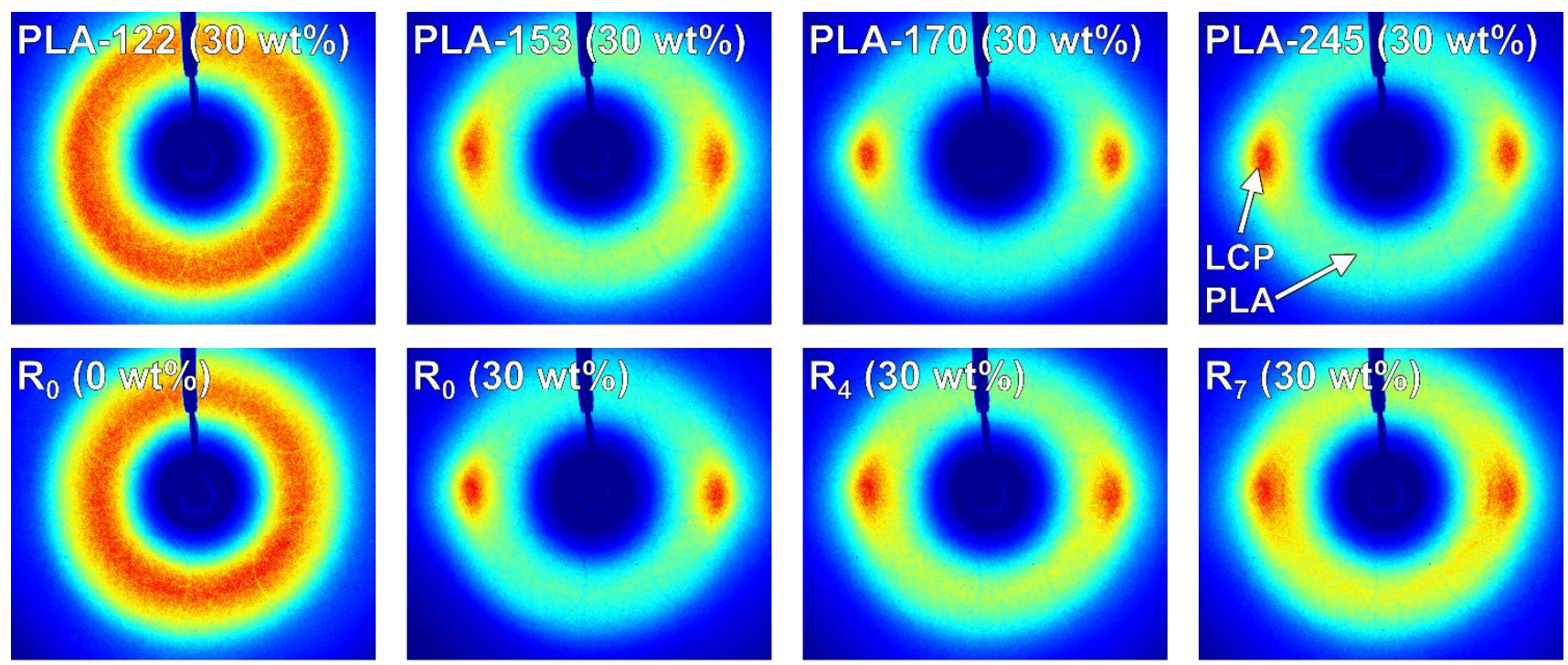

Min

Max

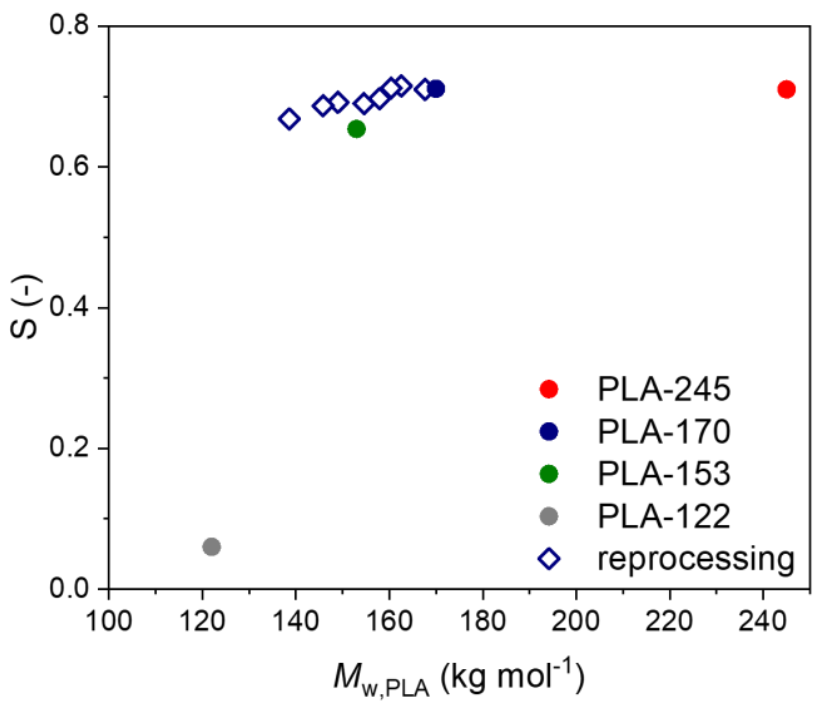

Figure S1. WAXD-diffractograms of several samples, flow direction is vertical in the image-plane (top). Orientation parameter $S$ as a function of matrix $M_{w}$, as calculated from the diffractograms. The orientation parameter was corresponds mainly to the degree of interchain orientation of the $L C P$, where $S=1$ signifies perfect alignment and $S$ = 0 signifies a completely isotropic state (bottom). 
The bottom figure shows the orientation parameter $\mathrm{S}$, signfying the degree of interchain orientation in the LCP, as a function of the molecular weight of the PLA matrix. At high PLA $M_{\mathrm{w}}$ the LCP is oriented. A decrease in $M_{\mathrm{w}}$ initially does not result in a change in S. Lowering $M_{\mathrm{w}}$ further, below $170 \mathrm{~kg} \mathrm{~mol}^{-1}$, leads to a gradual decrease in orientation, and at some point even a completely isotropic state. This is in accordance with the morphologies of the composites obtained via SEM and POM, and corresponds with the observed mechanical performance. This confirms that the LCP is oriented when the dispersed LCP droplets are deformed and stretched during processing.

Wide-angle X-ray diffraction (WAXD) measurements (wave length, $\lambda=0.104 \mathrm{~nm}$ ) were carried out at the European Synchrotron Radiation Facility (ESRF), the DUBBLE beamline (BM26B, Grenoble, France). The DUBBLE beamline is optimized for polymer science as is reported by Bras et al. ${ }^{1}$ and Portale et al. ${ }^{2}$. WAXD patterns, with an exposure time of $10 \mathrm{~s}$, were collected using a Frelon detector $(2048 \times 2048$ pixels of $48.8 \mu \mathrm{m} \times 48.8 \mu \mathrm{m})$ placed at a distance of $0.18 \mathrm{~m}$. The data were normalized for synchrotron beam fluctuations using an ionization chamber placed before the sample. Furthermore, a correction for the sample absorption was performed using a photodiode located at the beamstop before the background contribution was subtracted. The wavenumber $\mathrm{q}=$ $4 \pi \sin \theta / \lambda$, with $\theta$ being half of the scattering angle for WAXD experiments scale calibration, has been achieved by $\alpha-\mathrm{A} 12 \mathrm{O} 3$ (alumina).

The orientation parameter $(\mathrm{S}),\left\langle\mathrm{P}_{2 \mathrm{n}}(\cos \varphi)>_{\mathrm{d}}\right.$, was calculated from the obtained diffraction patterns via the procedure described by Mitchell and Windle ${ }^{3}$. The azimuthal intensity $\mathrm{I}(\varphi)$ at the maximum of the inter-chain diffraction peak $\left(2 \theta=21^{\circ}\right)$ was taken. The orientation parameter $<\mathrm{P}_{2 \mathrm{n}}(\cos \varphi)>_{\mathrm{d}}$ was then determined from an average of a Legendre polynomial, weighted against 
the obtained azimuthal intensity scan using equations (1) - (3). In this case, only the second order Legendre polynomial was taken into account, $\left\langle\mathrm{P}_{2 \mathrm{n}}(\cos \varphi)\right\rangle_{\mathrm{m}}=-0.5$.

$$
\begin{gathered}
\mathrm{S}=<\mathrm{P}_{2 n}(\cos \varphi)>_{d}=\frac{<\mathrm{P}_{2 n}(\cos \varphi)>}{<\mathrm{P}_{2 n}(\cos \varphi)>_{m}} \\
<\mathrm{P}_{2 n}(\cos \varphi)>=\frac{\int_{0}^{\frac{\pi}{2}} I(\theta, \varphi) \mathrm{P}_{2 n}(\cos \varphi) \sin \varphi \delta \varphi}{\int_{0}^{\frac{\pi}{2}} I(\theta, \varphi) \sin \varphi \delta \varphi} \\
<P_{2 n}(\cos \varphi)>_{m}=\frac{(2 n) !}{(-1)^{n} 2^{2 n}(n !)^{2}}=-\frac{1}{2} \text { for the second term }
\end{gathered}
$$

The obtained orientation parameter reflects the contributions of the distribution of the director orientation throughout the bulk poly-domain sample and the contributions of the director on a molecular level. In short, the orientation parameter reflects the degree of anisotropy of the scattering of polymer chains, while assuming that these chains are infinitely long rigid rods. The values of S vary from 0 (completely isotropic liquid state), to unity (perfect alignment of the polymer chains along the orientational axis).

(1) Bras, W.; Dolbnya, I. P.; Detollenaere, D.; Van Tol, R.; Malfois, M.; Greaves, G. N.; Ryan, A. J.; Heeley, E. Recent Experiments on a Combined Small-Angle/Wide-Angle X-Ray Scattering Beam Line at the ESRF. $J$. Appl. Crystallogr. 2003, 36 (3 I), 791-794. https://doi.org/10.1107/S002188980300400X.

(2) Portale, G.; Cavallo, D.; Alfonso, G. C.; Hermida-Merino, D.; Van Drongelen, M.; Balzano, L.; Peters, G. W. M.; Goossens, J. G. P.; Bras, W. Polymer Crystallization Studies under Processing-Relevant Conditions at the SAXS/WAXS DUBBLE Beamline at the ESRF. J. Appl. Crystallogr. 2013, 46 (6), 1681-1689. https://doi.org/10.1107/S0021889813027076.

(3) Mitchell, G. R.; Windle, A. H. Orientation in Liquid Crystal Polymers. In Developments in crystalline polymers; Basset, D. C., Ed.; Elsevier Applied Science: London, UK, 1988. https://doi.org/10.1007/978-94009-1341-7_3. 


\section{Complex viscosity of PLA-170 after each reprocessing cycle.}

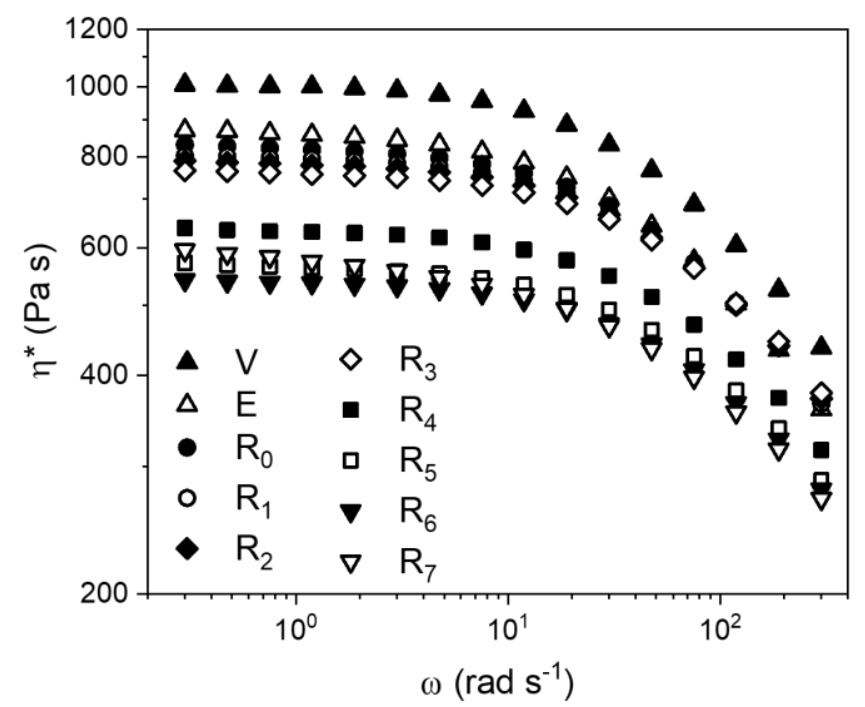

Figure S2. Complex viscosity as a function of oscillation frequency of PLA-170 (0 wt\% LCP) after each reprocessing cycle. $T=190^{\circ} \mathrm{C}$.

Figure S2 shows that the molecular weight of the PLA gradually decreases over the course of multiple reprocessing steps, as the zero-shear viscosity gradually decreases and the onset of shearthinning gradually shifts to higher frequencies. Conform the GPC data, rheometry incates no changes in the shape of the molecular weight distribution, with the exception of $\mathrm{R}_{7}$. After 7 reprocessing steps, a small deviation from this behavior is observed: at the low frequency side of the curve, there is a slight upturn in the viscosity after it had seemingly reached its plateau value $\left(\eta 0^{*}\right)$; this is an indication for either the formation of a small amount of long chain branches, or the presence of network of a particulate nature due to contamination resulting from reprocessing. 


\section{Stability of the LCP under the applied reprocessing conditions.}

The LCP was subjected elevated temperatures for a significant period in a plate-plate rheometer, in order to assess the likelihood of changes in the visco-elastic response over several reprocessing cycles (Figure S3). Assuming a residence time of 180 seconds at the processing temperature per cycle, the cumulative residence time is 2000 to 2500 seconds, corresponding to a minor variation in the LCP viscosity. It should be noted that changes might be accelerated under actual processing conditions, but a large change LCP viscosity over the course of several reprocessing cycles remains unlikely.

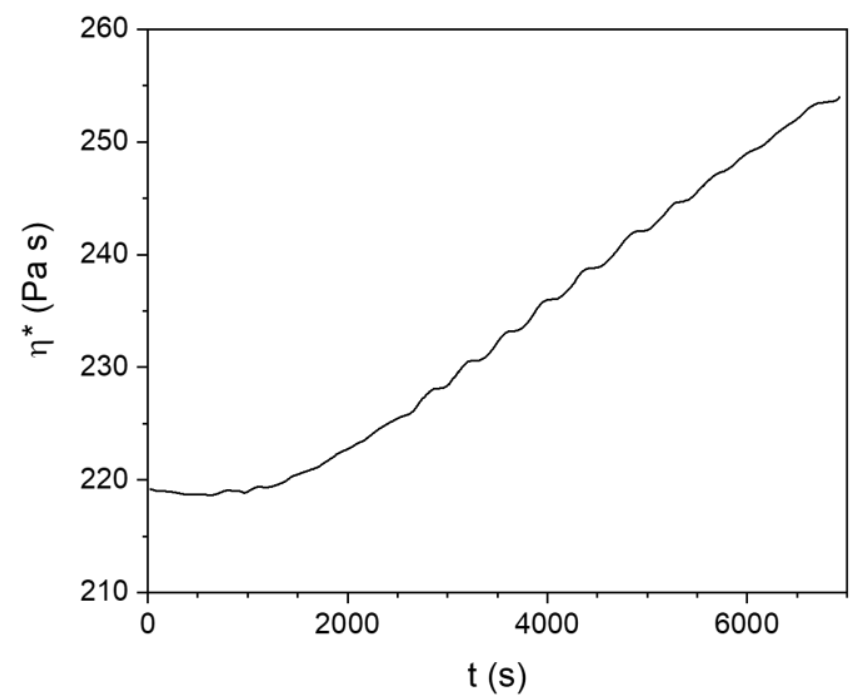

Figure S3. Complex viscosity of the thermotropic LCP as a function of time. $T=220^{\circ} \mathrm{C}, \omega=10 \mathrm{rad} \mathrm{s} \mathrm{s}^{-1}$.

To ensure that the viscoelastic behavior or the LCP remains similar upon reprocessing of the composites, the LCP was isolated and characterized after the last reprocessing step $\left(\mathrm{R}_{7}\right)$. The reprocessed LCP was recovered via dissolution of the PLA matrix in chlorform, followed by filtration and drying. Even though the LCP has been reprocessed 7 times, the viscoelstic response (Figure S4), especially in the high frequency regime, remained identical to that of the original material. Due to limitations of the applied filtration procedure, a small amount of PLA is still 
present in the reprocessed LCP. The observed differences in the viscoelastic behavoir at low frequencies, could be caused by the response of small dispersed PLA droplets. Therefore, changes in the flow behavior of the LCP are expected to have no effect on the morphological development.

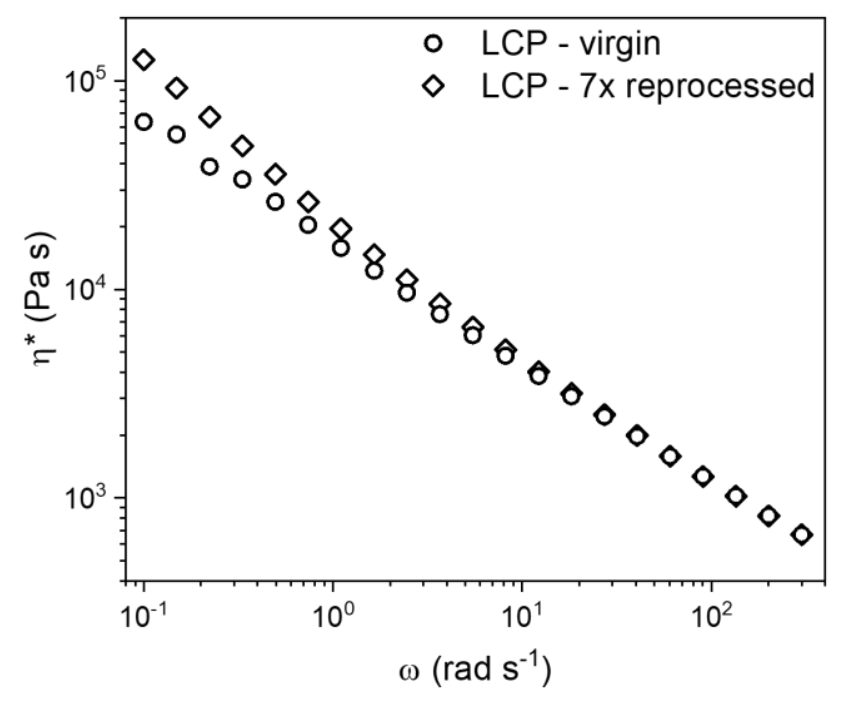

Figure S4. The complex viscosity of virgin LCP and LCP recovered after 7 reprocessing cycles plotted versus the angular frequency. Measured at $200^{\circ} \mathrm{C}$.

Note that there is a discrepancy between the viscoselstic response of the LCP reported here and elsewhere in this work. This is due to differences in the experimental set-up, as a different parallel plate set-up was used (plate diameter $=25 \mathrm{~mm}$, gap $=0.5 \mathrm{~mm}$, strain $=0.5 \%$ ). The viscoelastic response of LCPs is complex and highly sensitive to such changes, as it depends on the polydomain texture of the material during the actual measurement. 


\section{Development of the maximum tensile stress over the course of seven reprocessing cycles.}

The LCP proved to be effective in reinfrocing the PLA, as is confirmed by the increase in maximum stress in Figure S5. This effect was more significant at higher LCP-content, and persisted over the course of the seven reprocessing cycles. However, over the course of seven reprocessing cycles the maximum stress was found to drop. Decreases in stress of $28 \%, 15 \%$, and $18 \%$ were found for the materials containing $30 \mathrm{wt} \%, 10 \mathrm{wt} \%$, and $0 \mathrm{wt} \% \mathrm{LCP}$, respectively. For most samples, a relatively large decrease was observed over the course of the seventh cycle, as the drop in stress over the first six cycles corresponds to $18 \%, 11 \%$, and $11 \%$, respectively.

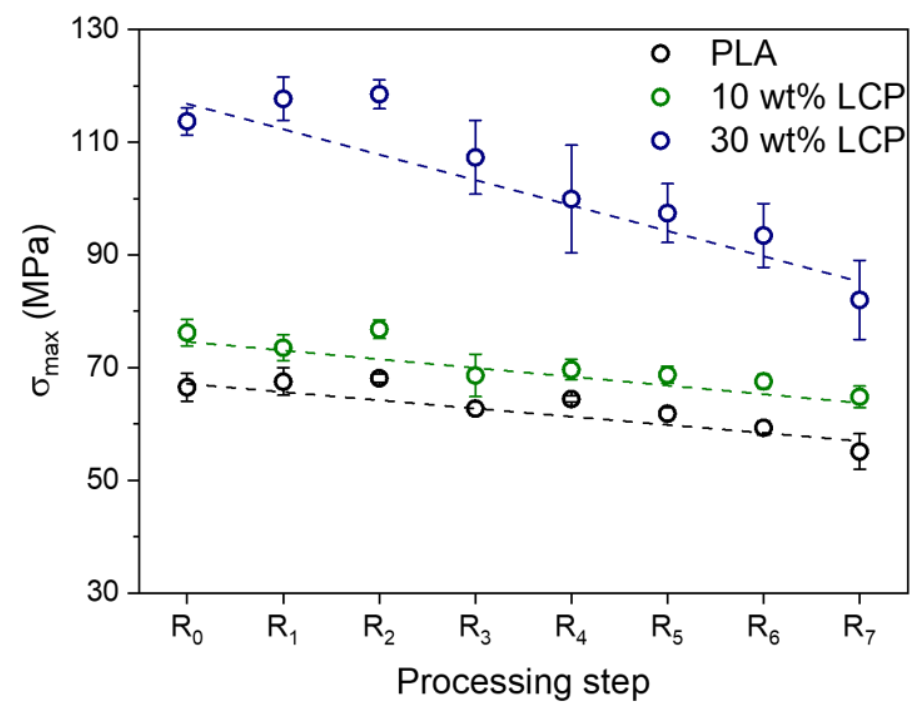

Figure S5. Development of the maximum tensile stress over the course of seven reprocessing cycles. The maximum stress corresponds to the stress at break in the case of PLA-LCP composites and to the yield stress in the case of pure PLA.

The yield stress is reported for the pure PLA, whereas the stress at break is reported for the PLALCP composites. This was done in order not to over-emphasize the reinforcing effect of the LCP. It was not possible to report the yield stress for all samples, as the PLA-LCP composites did not yield. 


\section{Themal behavior of LCP and PLA-170}

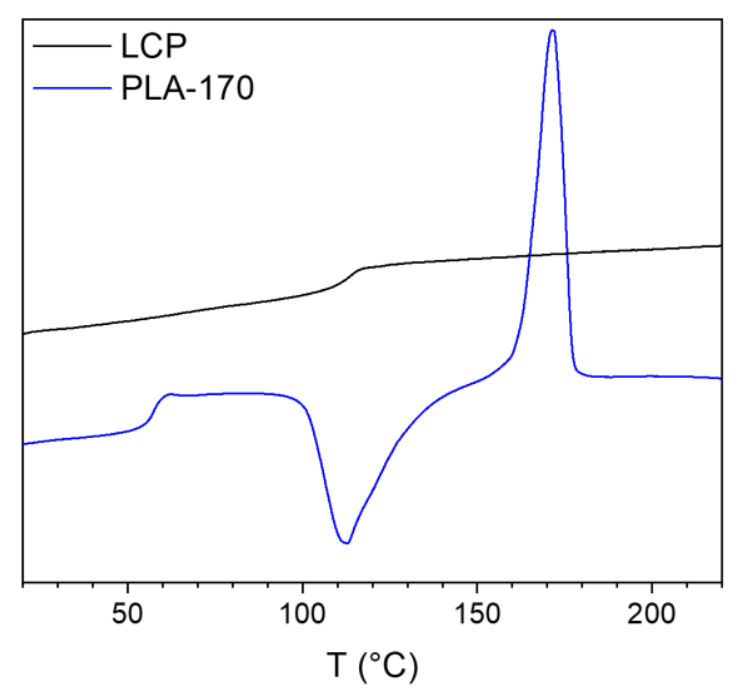

Figure S6 Differential scanning calorimetry thermograms of the second heating step of LCP and PLA-170, respectively. Measurements were carried out at a heating $10^{\circ} \mathrm{C} \mathrm{min}^{-1}$ (endo up).

The thermal behavior of LCP and PLA-170, measured via differential scanning calorimetry, are shown in Figure S6. LCP has a high $T_{\mathrm{g}}$ around $110^{\circ} \mathrm{C}$, and does not display a clear melting peak. This behavior is expected for a fully aromatic copolymer, which does not exhibit extensive formation of non-periodic layer crystallites. PLA-170 grade crystallizes slowly, as indicated by the presence of a large cold crystallization peak during heating. PLA-170 exhibits a $\mathrm{T}_{\mathrm{g}}$ around 60 ${ }^{\circ} \mathrm{C}$ and a peak melting temperature of $175^{\circ} \mathrm{C}$. 